$\begin{array}{ll}\text { Research Square } & \begin{array}{l}\text { Preprints are preliminary reports that have not undergone peer review. } \\ \text { They should not be considered conclusive, used to inform clinical practice, } \\ \text { or referenced by the media as validated information. }\end{array}\end{array}$

\title{
The Applicability of eVTOLs in Emergency Medical Care in Japan
}

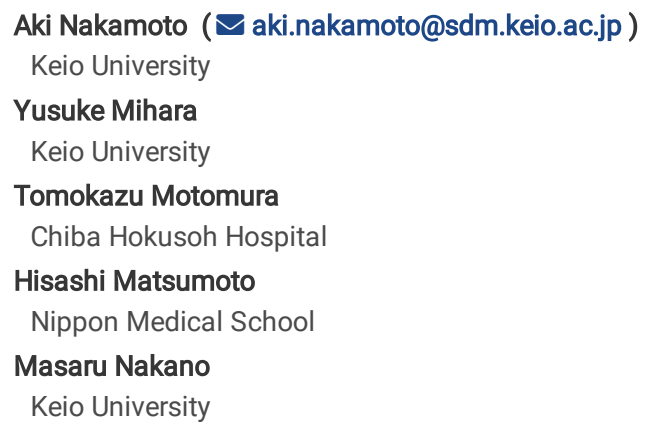

\section{Research Article}

Keywords: Emergency Medical Care, Electric Vertical Takeoff and Landing (eVTOL) Aaircraft, Physician-Staffed Emergency Medical Helicopters, Interview Survey, Flight Data Analysis

Posted Date: March 8th, 2022

DOI: https://doi.org/10.21203/rs.3.rs-1218578/v2

License: @) (7) This work is licensed under a Creative Commons Attribution 4.0 International License. Read Full License 


\section{Abstract}

Background: The present study aims to elucidate the applicability of electric vertical takeoff and landing (eVTOL) aircraft that dispatch only a doctor to provide a solution to the operational challenges of utilizing a helicopter to transport a doctor to the patient/s, with such physician-staffed emergency medical helicopters being known as doctor heli (DH), in Japan.

Methods: This study conducted interviews with 17 parties related to DHs in five prefectures in Japan to depict challenges of DHs and eVTOL requirements. Subsequently, this study analyzed the Hokusoh DH flight data, as an example, in terms of cases for which flight doctors consider condition assessment and initial treatment provision by a doctor would be particularly effective, to assess the hypothesis that a two-seater is applicable for emergency medical care (EMS) and that eVTOLs help reduce duplicate dispatch requests for DHs as well as the percentage and delayed dispatch time of duplicate requests.

Results: Challenges of DHs and eVTOL requirements were identified and classified into six major categories. Data analysis results indicated that two-seater eVTOLs would be particularly effective for trauma, cardiac disease, brain disease cases, and pediatric patient cases and help solve duplicate requests.

Conclusions: Two-seater eVTOLs are likely applicable in EMS in Japan.

\section{Introduction}

A helicopter to transport a physician to the intended location of the patient/s (usually a designated rendezvous point closest to the patient) is useful in Japanese helicopter emergency medical services (HEMS) since 2001 when operations started, with such physician-staffed emergency medical helicopters being known as doctor heli $(\mathrm{DH})$, in Japan. Nevertheless, it still presents some challenges. The golden hour is defined as $\leq 60$ min from the time a severe injury occurred; a lifesaving treatment should be provided during this period. ${ }^{1}$ Hence, $54 \mathrm{DH}$ are currently deployed to base hospitals in 45 prefectures in Japan. ${ }^{2}$ Each DH is generally staffed with one or two flight doctors, a flight nurse, a pilot, and a mechanic and transports a patient to the DH base hospital or another advanced critical care center after initial treatment in the field. The operational costs are funded via the taxation system in Japan like EMS in many countries in the world including Scandinavian countries and Canada. ${ }^{3}$ Helicopter transports are observed to be more effective than ground transport by ambulance. ${ }^{4}$ However, challenges remain for further DH development in Japan. The challenges are summarized into six points: (1) securing staff (e.g., an emergency physician), (2) introduction and operation costs, (3) inability to operate at night, (4) challenges with landing sites (DHs practically cannot land anywhere), (5) inability to dispatch a DH when it is being dispatched or when a simultaneous request is made, and (6) inability to fly in bad weather. ${ }^{5}$

Despite the situation, electric vertical takeoff and landing (eVTOL) aircraft, the so-called flying cars, are expected to be commercialized soon. eVTOLs are expected to be smaller, lighter, less expensive, less noisy, and safer than conventional helicopters and be unmanned and fly at night in the long term. ${ }^{6}$

Conventional studies consider "eVTOL with a total of five to eight passengers, including a patient and crews" 7 and "eVTOL with a total of four passengers, including a patient and crews" ${ }^{18}$ with the assumption of application in the United States. HEMS in the United States dispatch paramedics. However, HEMS in Japan dispatch a doctor to a patient to assess the clinical state and provide initial treatment in the field. ${ }^{9}$ This is due to the difference of the two main prehospital systems of deliverly ipmlemented in EMS: the Franco-German and the Anglo-American model ${ }^{10,11}$. In the fomer model employed by most of the European countries, physicians are usually accompanied by a nurse, a medical technician, or a paramedic, and sent to the scene, with a focus on diagnosis and treatment. This is the case also in Japan. In the latter model employed by the USA, Canada, the UK, Ireland, Australia, and most of the Middle East and South Africa, however, paramedics are sent to the scene as the highest level of ambulance-based clinicians, with a focus on prompt transport to hospital.

This approach is characterized by the active intervention by a doctor ${ }^{12}$ before the patient is transported to a hospital. The primary goal of the Japanese HEMS is early contact between a doctor and patient(s). Thus, the applicability of eVTOLs in Japanese emergency medical services (EMS) needs to be studied. Conventional studies include a comparison of the costs of three types of eVTOL airframes to be applied to the Japanese EMS. ${ }^{13}$ However, it focused on one of the challenges of DHs (i.e., the high cost) and did not elaborate on why eVTOLs are needed instead of helicopters considering the challenges of DHs other than the high costs.

This study, therefore, aims to elucidate the applicability of eVTOLs that dispatch only a doctor to provide a solution to operational challenges of DHs. Figure 1 shows various research topics required for realizing eVTOLs to be used in EMS. This study will present the challenges of DHs and address the reduction of duplicate requests.

This study comprises four sections. The Methods section will explain the flow and methods of the interview survey with DH-related parties in Japan and analysis of the Hokusoh DH flight data. The Results section will present the utility and challenges of DHs on the basis of the interview survey and the applicability of eVTOLs through the quantitative analysis. The Discussion section will summarize this study, interpret the results, and show future research topics.

\section{Methods}

\section{Interview survey with DH-related parties in Japan}

The interviews were conducted between October 2017 and November 2020 with 17 organizations involved in DHs in Japan (Fig. 2). This study selected several base hospitals located in urban and rural areas because the operation of each DH is characterized by the local medical needs, helicopter operation, medical resources and operations, and geographical conditions. The executive of a base hospital was an assistant director and his views on the management of the DH base hospital and the Japanese EMS scheme were solicited. 
This study conducted interviews with several DH operators providing HEMS in urban and rural areas. Besides pilots, mechanics, and communication specialists, the interviewees who work in the helicopter companies included an executive to solicit thoughts on the management of the company and the Japanese EMS scheme.

The Japanese Paramedics Association, a professional organization of paramedics, was interviewed to determine the needs of municipal ambulance and firefighting units, which are dispatched to sites with the patient(s) when a DH is dispatched. The private paramedic service company is commissioned to provide emergency rescue services in small-sized municipalities that do not provide permanent ambulance and firefighting services. The helicopter manufacturer is a Japanese manufacturer of DH airframes.

Patients and their families were excluded from the interviews because people whose lives were saved through the use of DHs are grateful for the rapid medical transportation and initial treatment. ${ }^{14}$ However, they assume a low priority for this study to elucidate the required specifications of the airframe and infrastructure.

The following were the common questions asked in each interview for each DH from the relevant party's perspectives: (1) the current status of the DH business (number of passengers, departure and landing points, consumed time, airframe and operational cost, geography and weather conditions, flight altitude, utilization rate [the annual percentage of DH flights that have successfully flown without cancellations, excluding those caused by inclement weather], and rules specific to the DH and others); (2) DH utility; (3) challenges in ensuring the DH utility and current measures for improvement; (4) transport challenges of $\mathrm{DH}$; and (5) the eVTOL requirements for resolving the challenges (number of passengers, flight operations, the use of the assumed eVTOL and existing HEMS transport methods, departure and landing points, expected time, payment amount, flight altitude, utilization rate, safety, autopilot, nighttime operation, and challenges to address realization).

\section{Analysis Of The Hokusoh Dh Flight Data}

The flight data of the Hokusoh DH in FY 2018 provided by Nippon Medical School, Chiba Hokusoh Hospital (hereinafter referred to as Hokusoh Hospital), will be used for the analysis.

Cases in which clinical state assessment and initial treatment provision by a doctor dispatched without other medical crews would be particularly useful

The flight doctors of the Hokusoh DH suggested the following during the interview: "Due to the limited payload of eVTOLs when the new aircraft is introduced in EMS, it is not necessary to carry medical equipment equivalent to that of a DH to the field at the stage. More equipment can be loaded as the payload increases in the technical advancement." "Clinical state assessment and initial treatment provision would be particularly effective for trauma, cardiac disease, brain disease cases, and pediatric patient cases."

With clinical state assessment and most initial treatment provisions being permitted to doctors only and not paramedics in Japan, conventional studies have yet to show in which cases clinical state assessment and initial treatment provision by a doctor are particularly useful. The conventional studies include an analysis of the cases of patients transported by a DH. ${ }^{15}$ Regarding the Hokusoh $\mathrm{DH}$, a study exists on the number of patients with stroke and the transport time for those who were transported by the $\mathrm{DH}$ and admitted to the hospital's Neuroscience Center. ${ }^{16}$ However, no study has shown in which cases it is particularly useful for doctors to make an early on-scene diagnosis about clinical conditions that cannot be made by paramedics.

Thus, this study will reveal the percentage of the aforementioned cases using the Hokusoh DH flight data ${ }^{17}$ annually compiled by the Japanese Society for Aeronautical Services from FY 2013 to 2017. Cardiac diseases include cardiovascular (acute coronary syndrome, aortic aneurysm/dissection, and so forth) and cerebrovascular (cerebral infarction, subarachnoid hemorrhage, cerebral hemorrhage, and so forth) diseases. Pediatric patient cases represent transport of patients aged 15 years old or under. Pediatric patient cases include both transport for operating in the field and interfacility transport, whereas trauma, cardiac disease, and brain disease cases include only the former.

Duplicate requests

This study defines a duplicate request as a case wherein another request for DH is made before the dispatched DH returns to the base hospital. This study analyzes the situation of duplicate requests for the Hokusoh DH in FY 2018. First, the present study indicates whether dispatch request duplication occurred or not (case with a duplicate request or case without a duplicate request). If duplication occurs, whether the Hokusoh DH was dispatched or not (dispatched or not dispatched) will be indicated. Subsequently, the percentage and delay time for cases wherein the dispatch of the latter case was delayed because of dispatch requests during the former dispatch will be shown. Delay is defined as the difference between the time of the dispatch request and the end time of the former case (landing at the Hokusoh Hospital, another hospital, or a rendezvous point). One case is assumed if one case contains multiple patients. A duplicate request is assumed if a request is simultaneously made.

\section{Results}

\section{Identifying the requirements for eVTOLs}

DH utilities, challenges in ensuring DH utilities, and current measures for improvement

On the basis of the interview survey results, the time-lapse between DH dispatch (from a DH request call to a DH base hospital to the landing of the DH at a receiving hospital) were divided into six slots and showed $\mathrm{DH}$ utilities, challenges in ensuring $\mathrm{DH}$ utilities, and current measures for improvement for each time 
slot (Table 1).

Table 1

DH utilities, challenges in ensuring DH utilities, and current measures for improvement perceived

DH utilities

$\begin{array}{ll}\text { Elapsed time } & \text { Rapid } \quad \text { Rapid transport of a patient } \\ & \text { dispatch } \\ & \text { of a } \\ \text { doctor }\end{array}$

\section{Emergency call}

to dispatch

request
Challenges in ensuring DH utilities, and current measures for improvement
Formation
of wide-
area
medical
zones

Taking off from

the DH base

hospital to

landing at a site

\section{Gathering infrequent and special cases}

\section{Measures: \\ - Progress has been made in the installation of D-Call Net system in cars to shorten the time between the occurrence of a traffic accident and the request for a $\mathrm{DH}$. The introduction of} the system has been largely completed at base hospitals.

- A potential exists for improving the quality of emergency calls made by citizens using a smartphone video transmission system.

Challenges:

- Delay in dispatch when duplicate requests are made.

- Contacting the patient(s) may take time due to waiting in the air until a firefighting unit arrives.

Measure:

- The introduction of new or additional DH and the development of high-speed helicopters (all of which are expensive in terms of aircraft and operation).

Challenge:

- Stabilizing the patient before takeoff from the site takes time in the case of long-distance transportation in Hokkaido, the second-largest and northernmost of Japan's four main

islands, or remote islands.

$\begin{array}{ll}\begin{array}{l}\text { Taking off from } \\ \text { the site to } \\ \text { landing at a } \\ \text { receiving } \\ \text { hospital }\end{array} & \begin{array}{l}\text { Shortening the time required for patient } \\ \text { transport (including transport to an } \\ \text { advanced critical care center and } \\ \text { interfacility transport in medically } \\ \text { underserved areas) }\end{array} \\ & \begin{array}{l}\text { Preventing ambulances from being } \\ \text { unavailable for long periods in medically } \\ \text { underserved areas }\end{array} \\ \begin{array}{l}\text { Landing at the } \\ \text { receiving } \\ \text { hospital }\end{array} & \begin{array}{l}\text { Earlier definitive treatment initiation, } \\ \text { thereby higher potential for medical } \\ \text { economic benefit than ground ambulance } \\ \text { transport }\end{array}\end{array}$

Landing at the

patient[s]) to

taking off from

the site

The DH utilities can be grouped into rapid dispatch of a doctor, rapid transport of a patient, and formation of wide-area medical zones. For rapid dispatch of a doctor, DHs are likely to enhance the lifesaving rate by dispatching a doctor to a patient as soon as possible and providing initial treatment. For patient transport, DHs are likely to speed up the initiation of definitive treatment, including surgery, by transporting a patient to a hospital by a high-speed helicopter. Another advantage of patient transport is preventing ground ambulances from being unavailable for long periods in medically underserved areas. For the formation of a wide-area medical care system, DHs form wide-area medical care zones by gathering infrequent and special cases to $\mathrm{DH}$-based hospitals by taking advantage of the mobility and wide-area operation of helicopters and medical care systems that use helicopters. This is in line with the interview of this study with personnel of a private paramedic service company in the mountainous area of Kyushu, Japan's third-largest island located southwest of the main island Honshu. Referring to local patients, who are sometimes transported by $\mathrm{DH}$, the personnel commented that the helicopters are greatly helpful in terms of preserving ambulances because they do not have to spend $2-3 \mathrm{~h}$ transporting a patient by ground ambulance to an urban hospital. The DH utilities aforementioned is consistent with that of the conventional study. ${ }^{18}$

Next, on the basis of the interviews, this study will summarize DH utilities and current measures for improvement. Although measures for improvement have been observed in dispatching a DH to shorten the relevant time and increase efficiency and effectiveness from a DH request to takeoff from the base, ${ }^{19}$ reducing the time from taking off from a base to landing in the field is still a challenge. Possible measures could include the introduction of new/additional DHs or the development of high-speed helicopters. However, all of these have the challenge of high airframe and operational costs.

In the interview, the challenges of delays in dispatching a DH when a request duplication exists were also pointed out. A flight doctor from Red Cross Asahikawa Hospital said, "Duplicate requests account for $20 \%-25 \%$ of the reasons for non-dispatch. Duplicate requests occur in the case of long-distance missions, such as $300 \mathrm{~km}$ in a straight line or a two-hour round trip." A communication specialist at Hokusoh Hospital said, "When the weather is clear, 
duplicate requests often occur once a day, and it is common for two or three requests to overlap. Duplicate requests are common in the case of long-distance missions."

The challenge of waiting in the air until a firefighting unit arrives at the scene, which is usually a designated rendezvous point closest to the patient, was also pointed out. According to a flight doctor at Hokusoh Hospital, "In order for a DH to land, according to the prefectural government's guidance, the ground support team of the firefighting unit must ensure safety on the ground. If the arrival of the support team is delayed, the DH will have to wait in the air while circling, and the landing will also be delayed." The delay in reaching the patient is recognized as a challenge. Nevertheless, waiting in the air is not a major challenge in rural areas. A flight doctor at Red Cross Asahikawa Hospital said, "The good thing about rural areas is that you can land at almost any places. If the pilot decides that it is possible to land, he can get permission from the owner, ask the firefighting unit to ensure safety on the ground, and land on a road, private property, or farmland."

Thus, this study considers that the utility of eVTOLs for EMS as new mobility is shortening the time between taking off from the base and landing in the field. This could lead to earlier medical intervention by doctors, hospital selection, and treatment preparation at the receiving hospital, thereby leading to the concept of a two-seater eVTOL. Only a doctor would fly to the field by a piloted eVTOL, and a ground ambulance transports the patient to a hospital, contrary to a sixor seven-seater DH staffed with medical personnel and flight crews including a doctor and a pilot to transport a patient.

Challenges of DHs, requirements for resolving the challenges, and applicability of eVTOLs

Table 2 presents the challenges of DHs for the interviewed parties, the requirements for resolving the challenges, and the applicability of eVTOLs. The challenges were classified into six major categories on the basis of the interview results.

The first challenge is the cost. The high cost of helicopters, both in terms of airframe and operation, is a factor preventing the introduction of new DHs in prefectures where they have not yet been introduced. In prefectures wherein DHs have already been introduced, introducing additional helicopters is difficult, thus resulting in duplicate requests. Additionally, an executive at a DH base hospital said that high-cost DHs are unsustainable and that low-cost eVTOLs are likely key to the continuation of the air ambulance scheme in Japan.

The second challenge is the shortage of pilots. A shortage of helicopter pilots may occur in the future because of workforce aging. Operators are concerned about the possible difficulty of recruiting and hiring pilots and the deterioration of the working environment. Consequently, flight doctors are concerned that the skill level of pilots will decrease and that the safety of flight operations will deteriorate. Hence, promoting the automation of eVTOLs using technology, including sensors, is needed for easier operation, easier obtaining of licenses, and improved safety.

Table 2

\section{Challenges of DHs, requirements for resolving the challenges, and the applicability of eVTOLs}

\begin{tabular}{|c|c|c|}
\hline Challenges of DHs & \begin{tabular}{|c|}
$\begin{array}{c}\text { Requirements for resolving } \\
\text { the challenges }\end{array}$ \\
\end{tabular} & Applicability of eVTOLs \\
\hline $\begin{array}{l}\text { High cost of airframe and } \\
\text { operation, resulting in the } \\
\text { difficulty of introducing } \\
\text { additional helicopters and } \\
\text { duplicate re quests for } \\
\text { dispatch }\end{array}$ & Reduced cost & $\begin{array}{l}\text { Reducing the cost of airframe } \\
\text { and operation through } \\
\text { electrification and increased } \\
\text { efficiency in operation with } \\
\text { more aircrafts. }\end{array}$ \\
\hline Shortage of pilots & $\begin{array}{l}\text { Easy to operate and obtain a } \\
\text { pilot license }\end{array}$ & $\begin{array}{l}\text { Promoting the auto mation of } \\
\text { eVT OLs for easier operation, } \\
\text { o btaining licenses, and } \\
\text { improving safety. }\end{array}$ \\
\hline Large airframe size & $\begin{array}{l}\text { Increasing the number of } \\
\text { landing sites }\end{array}$ & $\begin{array}{l}\text { The small size of the airframe } \\
\text { and the capability to run on the } \\
\text { ground if possible would allow } \\
\text { eVT OLs to land at the point } \\
\text { where it is not possible for a } \\
\text { helicopter to land and also } \\
\text { dire ctly access the patient(s) in } \\
\text { the field. }\end{array}$ \\
\hline Large downwash amount & Reduced downwash amount & $\begin{array}{l}\text { The smaller size of the airframe } \\
\text { would allow a reduction in the } \\
\text { downwash amount. }\end{array}$ \\
\hline High noise level & Reduced noise level & $\begin{array}{l}\text { The smaller size of the airframe } \\
\text { would allow a reduction in the } \\
\text { noise level. }\end{array}$ \\
\hline Inability to operate at night & Capability to operate at night & $\begin{array}{l}\text { With safe auto pilot technology, } \\
\text { increasing the feasibility of } \\
\text { nighttime operations. }\end{array}$ \\
\hline
\end{tabular}

(Note)

DHs: DH denotes a helicopter to transport a physician to the intended location of the patient/s (usually a designated rendezvous point closest to the patient), which is used in Japanese helicopter

emer gency medical services (HEMS) since 2001, with such helicopters being known as doctor heli

$(\mathrm{DH})$

eVTOLS: electric vertical takeoff and landing (eVTOL) aircraft

The third challenge is the large airframe size. Several instances showed that the large size made it impossible for DHs to directly access patient(s) in the field, including accident sites; hence, preventable mortality occurred. This has been recognized by medical personnel, operation personnel, and the prefectural 
government as a concern. An executive of an operator said, "It is crucial how freely eVTOLs can select a place to take off and land. Otherwise, the difference between eVTOLs and helicopters will not be clear, and they will not be useful enough to use, which is the same environment as the current helicopter industry in Japan. Takeoff and landing site requirements should also be separated from those for current helicopters so that they can be set more easily." Vertical taking off and landing with a smaller aircraft than helicopters, a significant increase in takeoff and landing sites, and more flexible operation are expected

If eVTOLs are compact and can run on the ground, they can directly access places wherein helicopters have difficulty landing or reaching patient(s). For example, the Hokusoh DH (MD902) has a length, width (main rotor blade diameter) and requires takeoff and landing area of $12.37 \mathrm{~m}, 10.34 \mathrm{~m}$, and at least $35 \mathrm{~m}^{2}, 20$ respectively, which limits the number of takeoff and landing sites. This is one of the reasons why it is frequently challenging to quickly reach patient(s) near the scene. By contrast, an eVTOL, such as EHang 184, ${ }^{21}$ which measures $3.86 \times 5.5 \mathrm{~m}$, is likely to land in a small space of approximately $6.5 \mathrm{~m}^{2}$. This assumes that the area required for a landing strip for an eVTOL is 1.2 times the size of the airframe (Article 79-8 of the Japanese Ordinance for Enforcement of the Civil Aeronautics Act under Article 36 of the Japanese Civil Aeronautics Act).

The fourth challenge is the large downwash amount. Therefore, landing sites are mainly limited to rendezvous points, and preventable mortality occurs. This is also recognized as a challenge by medical personnel, operation personnel, and the prefectural government. Water sprinkling is necessary to reduce the risk of brownouts. ${ }^{22}$ Moreover, the operators and prefectural government may receive complaints from nearby residents because of dust. Consequently, the relevant rendezvous point will become unusable if complaints are not resolved. eVTOLs may be able to reduce downwash using the compact airframe.

The fifth challenge is the high noise level. Hence, landing sites are mainly limited to rendezvous points; thus, preventable mortality occurs. This is also recognized as a challenge by medical personnel, operation personnel, and the prefectural government. According to Volocopter's whitepaper, the R22 helicopter emits $82 \mathrm{~dB}(\mathrm{~A})$ when hovering at an altitude of $75 \mathrm{~m} .{ }^{23}$ Conversely, Volocopter's multirotor eVTOL targets a low noise level of 65 and $76 \mathrm{~dB}(\mathrm{~A})$ at an altitude of 75 and $30 \mathrm{~m}$, respectively. ${ }^{23}$

The sixth challenge is the inability to operate at night. According to flight doctors, for example, approximately half of the total number of $\mathrm{DH}$ requests is made at night for the Hokusoh DH, which is not handled by the helicopter. On weekdays, rapid response cars are used to respond to patients from the end of the helicopter standby to 11:00 PM. However, they aim to reach the patient(s) by air also at night through nighttime operations. In response to this, according to the interview with the Medical System Development Office, Medical Development Division, Health and Welfare Department of the Chiba Prefecture, a working group had considered the idea in the past. However, nighttime operations were not realized because of cost, safety, and noise. For eVTOLs, autopilot technology could increase the feasibility of nighttime operations. ${ }^{24}$ However, to realize nighttime operations, enhancing the night response system of operators and improving night lighting and automatic guidance at the takeoff and landing sites are necessary, which present another challenge.

The interview survey of this study with the DH-related parties confirmed the applicability of a two-seater eVTOL. Additionally, this study considered that duplicate request is a challenge with the highest priority among the six challenges. For nonresponse due to duplicate requests, flight doctors, pilots, and communication specialists from all the hospitals interviewed are collaborating to avoid nonresponse as much as possible to save the lives that can be saved, which is a crucial concern. Other challenges are difficult to solve at this point with the current helicopter airframe and DH operations. Although efforts to solve these challenges were noted, they were considered to be of lower priority. Next, this study derived two research questions (RQs) on the application of eVTOLs in EMS to conduct a quantitative analysis on two points: the applicability of eVTOLs and the reduction of duplicate requests.

RQ1: Will a two-seater eVTOL be sufficient in terms of the number of passengers?

RQ2: Can a two-seater eVTOL help reduce duplicate requests?

These two RQs will be quantitatively analyzed in the next section using the Hokusoh DH flight data.

\section{Quantitative analysis of the applicability of eVTOLs}

Cases in which clinical state assessment and initial treatment provision by a dispatched doctor without other medical crews would be particularly useful

First, the results of the analysis are shown by cases in Table 3, which shows the number and percentage of trauma, cardiac disease, and brain disease cases as well as pediatric patient cases treated by the Hokusoh DH between FY 2013 and 2017.

Trauma, cardiac disease, and brain diseases as well as pediatric cases accounted for $72.4 \%-85 \%$ and $7.1 \%-10 \%$, respectively, over 5 years.

\section{Table 3}

Number and percentage of patients in the Hokusoh DH dispatches (FY 2013-2017) 


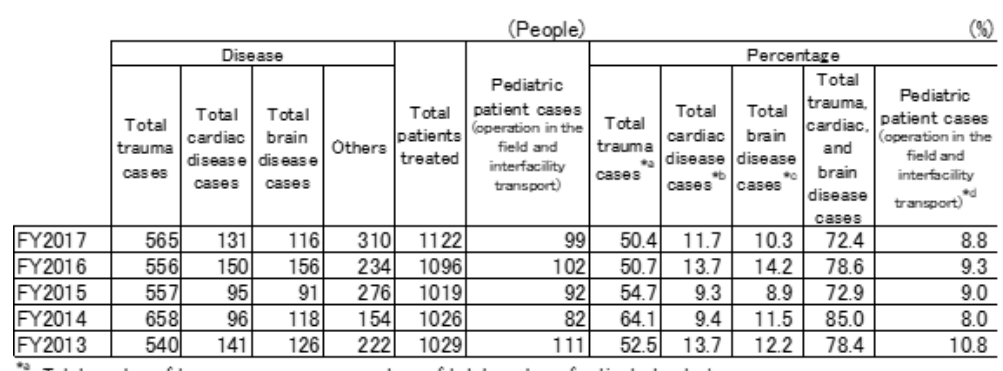

Total number of trauma cases as a percentaze of total number of patients treated

*t Total number of cardiac disease cases as a percentage of total number of patients treated

* Total number of brain disease casec as a percentage of total number of patients treated

* Total number of pediatric patient cases as a percentage of total number of patients treated

Duplicate requests

Figure 3 shows the percentage of duplicate requests for the Hokusoh DH in FY 2018. Of the 2,091 requests for dispatch, Hokusoh DH was dispatched and not dispatched in 1,229 and 862 cases, respectively. Moreover, 412 were requests during the dispatch of a previous case or duplicate requests. Of the duplicate requests, 141 were responded to by the Hokusoh $\mathrm{DH}$, although 271 were not, and the following actions were implemented. In 98, 52, 5, 10, and 106 cases, another DH was dispatched either from Kimitsu or Ibaraki, a ground ambulance transported the patients, the Hokusoh rapid response car was dispatched, other responses were taken including such cases that the ambulance unit was asked to reassess the condition after it arrived at the site, and the response was unknown, respectively.

Additionally, delays occurred in 65 cases in dispatching to the next case of the 141 cases wherein the Hokusoh DH was dispatched at the time of a duplicate request. Figure 4 shows that the minimum, maximum, mean, and median delays were 1, 45, 8.5, and 7 min, respectively. Moreover, the standard deviation was 7.6. Practically, no delay was noted in dispatching the $\mathrm{DH}$ to the next case for the rest of the 76 cases with a duplicate request. The following cases were included: a case in which a request for the next case was received while the $\mathrm{DH}$ is still in the field for the previous case and the doctor in charge of communication at the hospital decided to transport the previous patient by ground ambulance while the DH responded to the next case; after transporting the patient of the previous case to a hospital other than the base hospital, the next dispatch was requested, and the DH directly responded to the next case from the previous case; and a case in which the next case was requested while the $\mathrm{DH}$ was returning to the base from the previous case and the $\mathrm{DH}$ directly headed to the next case.

\section{Discussion}

The results shown in Table 3 indicate that trauma, cardiac disease, and brain disease as well as pediatric patient cases, for which flight doctors consider condition assessment and initial treatment provision by a doctor would be particularly effective, accounted for an average of $77 \%$ and $9 \%$, respectively, of all Hokusoh DH dispatches from FY 2013 to FY 2017. By dispatching doctors by eVTOLs, the survival rate of cases, as aforementioned, for which early clinical state assessments and initial treatment provision by doctors is useful, could be improved and the lives of patients may be saved even more.

The results shown in Figs. 3 and 4 indicate that the aforementioned delays in DHs could be solved by supplementing them with eVTOLs. With the introduction of eVTOLs, eVTOLs can likely be dispatched to cases that would be otherwise handled by another DH of a base hospital. However, careful examination of the cruise range is necessary. For example, Volocity, a fully electric, two-seater eVTOL, has a maximum range of $35 \mathrm{~km}$ feasible today. ${ }^{25} \mathrm{Moreover}$, the number of airframes required for an eVTOL needs to be estimated depending on the performance of the airframe (i.e., range and flight speed) and where and how to be located.

In conclusion, using a combination of interview methods with 17 parties related to DHs in five prefectures in Japan and analysis of the Hokusoh DH flight data, as an example, this study presented the following results:

1. Challenges of DHs and eVTOL requirements were identified and classified into six major categories.

2. Two-seater eVTOLs would be particularly effective for trauma, cardiac disease, and brain disease cases as well as pediatric patient cases and help solve the challenge of duplicate requests for $\mathrm{DHs}$.

This study thus concludes that two-seater eVTOLs for a flight doctor and a pilot are likely applicable in emergency medical care in Japan. Future research includes the quantitative estimation of the medical and economic effects of the operation of dispatching only a doctor to the patient(s). Another future research topic is to analyze the requirements for eVTOLs to land near a patient, for which the current DHs frequently have difficulty, and those for nighttime operations.

\section{Abbreviations}

$\mathrm{DH}$

Doctor helicopter (physician-staffed emergency medical helicopter in Japan)

EMS

Emergency medical care

eVTOL 
Electric vertical takeoff and landing

HEMS

Helicopter emergency medical services, RQs:Research questions.

\section{Declarations}

\section{Ethics approval and consent to participate}

The study was approved by the Nippon Medical School Chiba Hokusoh Hospital Ethics Committee (March 7, 2017). Written informed consent was obtained from patients for utilizing the data related to thier medical treatment. All methods were carried out in accordance with relevant guidelines and regulations. For the interviews with the personnel involved in the Japanese doctor heli, participation was voluntary. The Nippon Medical School Chiba Hokusoh Hospital Ethics Committee gave the approval to verbal informed consent, and thus verbalconsent was obtained in advance. No patients were included in the interviews. The subjects were only medical personnel including flight doctors and medical control doctors, in addition to industry personnel including helicopter operators, a helicopter manufacturer, a helipad construction company, and a private paramedic service company, as well as municipality and association personnel.

\section{Consent for publication}

Not applicable.

\section{Availability of data and materials}

The data analysed/used for the study would be availaible from the corresponding author (Aki Nakamoto) on reasonable request. The data that support the findings of this study are available from Shock and Trauma Center Hokusoh HEMS, Nippon Medical School Chiba Hokusoh Hospital, but restrictions apply to the availability of these data, which were used under license for the current study, and so are not publicly available.

\section{Competing interests}

The authors declare that they have no conflicting interests.

\section{Funding}

This work was supported by Keio University Doctoral Student Grant-in-Aid Program.

\section{Authors' contributions}

AN was responsible for conception and design of this study, as well as conducting interview surveys and data analysis, and contributed to drafting the manuscript. YM contributed to reviewing and editing the manuscript.TM and HM contributed to providing study resources and validation, and as well as reviewing and editing the manuscript. MN supervised this study. The authors read and approved the final manuscript.

\section{Acknowledgements}

The authors are grateful to Mr. Hideki Yokota of Aero Asahi for helpful discussions and comments.

\section{References}

1. Cowley RA, Hudson F, et al. An economical and proved Helicopter program for transporting the emergency critically ill and injured patient in Maryland. J Trauma. 1973;12:1029-38.

2. HEM-Net [in Japanese]. https://hemnet.jp/know-base. Accessed Jec 202022.

3. Bhandari D, Yadav NK. Developing an integrated emergency medical services in a low-income country like Nepal: a concept paper. Int J of Emerg Med. 2020:13:7.

4. Mashiko K [in Japanese]. Research on the actual circumstances and evaluation of "doctor helicopter." Grant-in-Aid for Research on Health and Labor Sciences, Study on the evaluation of new functions of medical emergency facilities and prehospital care system. 2016.

5. Kato K, Kuwatsuka K, et al [in Japanese]. Expansion of the "15-minute access area" by introducing a doctor helicopter: A memorandum for understanding the effect in consideration of operational constraints. Kokushikan University Geography Report. 2013;21:1-16.

6. Nakamoto A, Unno K, et al [in Japanese]. Market research study of flying cars in Hokkaido. In Proceedings of the 57th Airplane Symposium. 2019.

7. Booz Allen Hamilton. Executive briefing urban air mobility (UAM) market study, 2018. https://www.nasa.gov/sites/default/files/atoms/files/bah_uam_executive_briefing_181005_tagged.pdf. Accessed Jan 202022.

8. Chappelle C, Li C, et al. Opportunities to enhance air emergency medical service scale through new vehicles and operations. In Proceedings of the 18 th AIAA Aviation Technology, Integration, and Operations Conference 2018. 2018;233-248.

9. Yamada N, Kitagawa Y, et al. Validity and risk factor analysis for helicopter emergency medical services in Japan: a pilot study. BMC Emerg Med. 2021;21:87.

10. Makrides T, Ross L, et al. Exploring the structure and characteristics of the Anglo-American paramedic system in developed countries: a scoping review. Intl $\mathrm{J}$ of Emerg Serv. 2021 
11. Garrone M. Prehospital ultrasound as the evolution of the Franco-German model of prehospital EMS. Critical Ultrasound J. 2011;3:141-147.

12. Matsumoto $\mathrm{H}$ [in Japanese]. Future vision of emergency and disaster medical care system from the viewpoint of prehospital emergency care. J of Nippon Medical School. 2009;5:4:187-192.

13. Mihara Y, Pawnlada P, et al. Cost analysis of eVTOL configuration design for an air ambulances system in Japan. In Proceedings of the CESUN Conference 3. 2021.

14. HEM-Net [in Japanese]. Frontline of doctor helicopters. HEM-Net Graph No. 44, 2017.07.03. https://hemnet.jp/hem-net44. Accessed Jan 20 2022.

15. Ogura K, Yamakawa Y, et al [in Japanese]. Medical economic effects after the introduction of a doctor helicopter in Toyama Prefecture. J of Japanese Society for Aeromedical Services. 2018;19:1:18-25.

16. Kumagai T, Mishina M, et al. Use of emergency medical helicopters in stroke treatment in Chiba Prefecture. Stroke. 2008;30:4:545-550.

17. Kawasaki Medical School Hospital [in Japanese]. Report of Doctor Helicopter Operation Results for FY 2018. 2018.

18. Shock and Trauma Center Hokusoh HEMS, Nippon Medical School Chiba Hokusoh Hospital [in Japanese]. Hokusoh HEMS standard operating procedure. Herusu Shuppan, Tokyo, 2018. p. 2-3.

19. Motomura T, Matsumoto $\mathrm{H}$, et al [in Japanese]. Development and testing of advanced automatic collision notification; AACN (D-Call Net) to activate doctor-helicopter in Japan. $J$ of Japanese Society for Emergency Medicine. 2018;21:513-8.

20. Ministry of Land, Infrastructure, Transport and Tourism [in Japanese]. Administrative standards for permission at regional aviation bureaus for takeoff and landing at off airfield landing site. 1997.

21. eVTOL News. EHang doubles its 184. August 17, 2019. https://evtol.news/news/ehang-doubles-its-184. Accessed Jan 202022.

22. Yoshinaga $T$ [in Japanese]. Issues surrounding the safe operation of doctor helicopters. HEM-Net Symposium Report. 2015. https://www.hemnet.jp/databank/file/\%E3\%82\%B7\%E3\%83\%B3\%E3\%83\%9D\%E3\%82\%B8\%E3\%82\%A6\%E3\%83\%A0\%E5\%A0\%B1\%E5\%91\%8A\%E6\%9B\%B Accessed Jan 202022.

23. Volocopter $\mathrm{GmbH}$. Pioneering the urban air taxi revolution, 1.0. 2019. https://www.volocopter.com/content/uploads/Volocopter-WhitePaper-1-01.pdf. Accessed Jan 202022.

24. Read B. Flying taxis in the dark. May 24, 2019. https://www.aerosociety.com/news/flying-taxis-in-the-dark/?fbclid=IwAR2AZnooffv6Cz2 TJZReVdVG0jMw-5_bSioQAvbFMFmEaK1E-W3v0EkYzrQ. Accessed Jan 202022.

25. Volocopter GmbH. The Roadmap to Scalable Urban Air Mobility, White paper 2.0. 2021. https://www.volocopter.com/content/uploads/20210324_Volocopter_WhitePaper_Roadmap_to_scalable_UAM_m.pdf. Accessed Jan 202022.

\section{Figures}

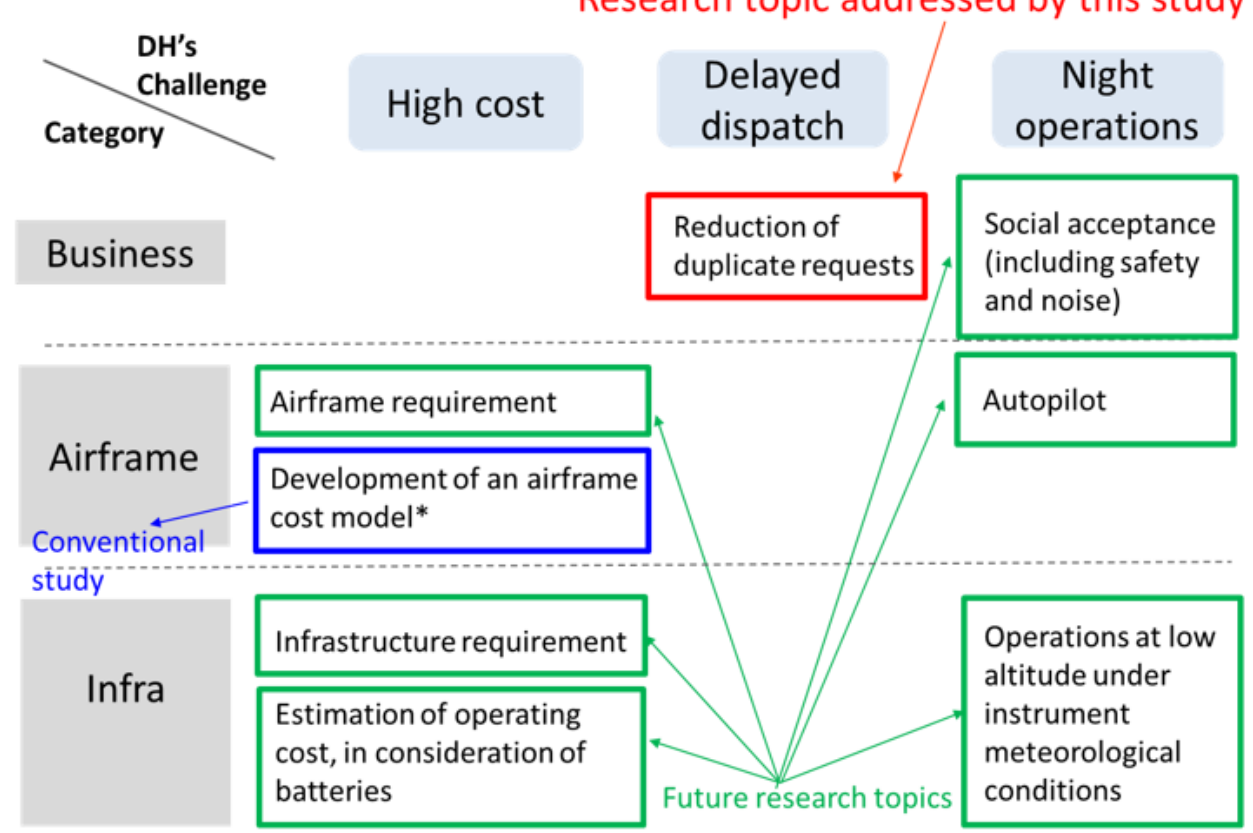

$\mathrm{DH}$ denotes a helicopter to transport a physician to the intended location of the patient/s (usually a designated rendezvous point closest to the patient), which is used in Japanese helicopter emergency medical services (HEMS) since 2001, with such helicopters being known as doctor heli (DH)

${ }^{*}$ Mihara Y, Pawnlada P, et al. Cost analysis of eVTOL configuration design for an air ambulances system in Japan. In Proceedings of the CESUN Conference 3. 2021. 
Research topics for eVTOLs to be used in EMS and the topics this study addresses.
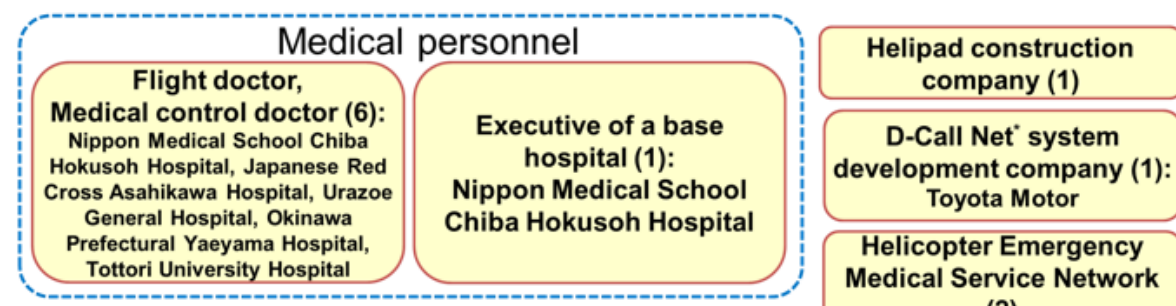

Helicopter Emergency Medical Service Network
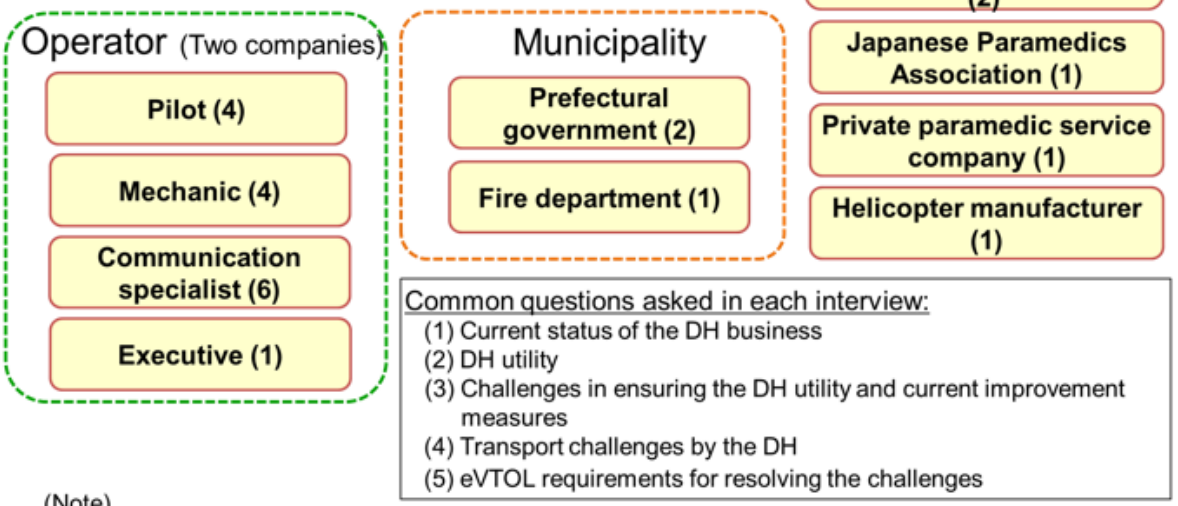

(Note)

(5) eVTOL requirements for resolving the challenges

The number in parenthesis shows the number of interviewees.

$\mathrm{DH}$ denotes a helicopter to transport a physician to the intended location of the patient/s (usually a designated rendezvous point closest to the patient), which is used in Japanese helicopter emergency medical services (HEMS) since 2001, with such helicopters being known as doctor heli (DH).

* D-Call Net is an advanced automatic collision notification (AACN) system. An event data recorder (EDR) equipped on a car automatically requests a $\mathrm{DH}$ dispatch in the event of a traffic accident.

${ }^{* \star}$ Helicopter Emergency Medical Service Network (HEM-Net) is a nonprofit organization that aims to promote physician onboarding emergency helicopters in Japan.

Figure 2

Interview subjects.

Case with a duplicate request:

412 cases

$19.7 \%$

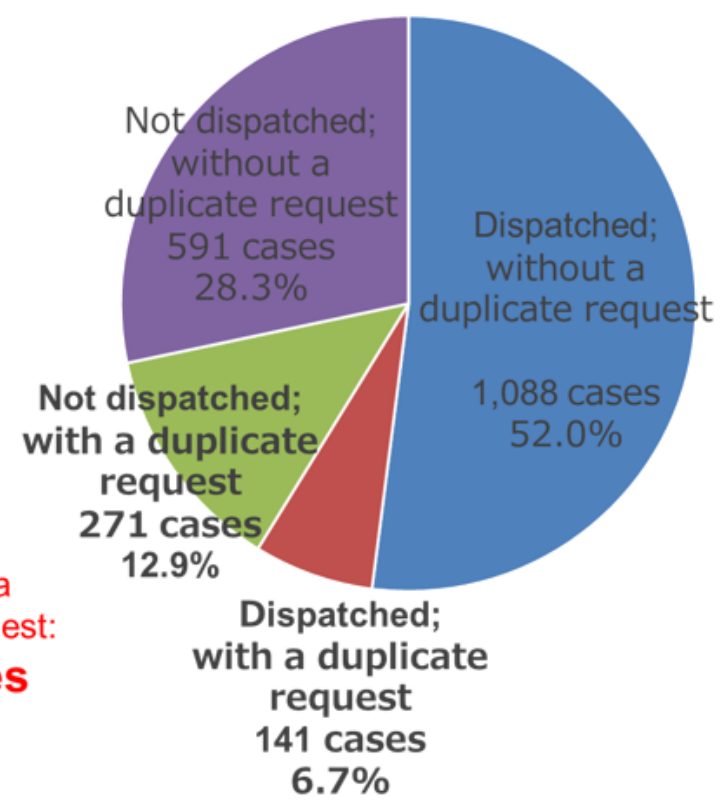

\section{A total of 2,091 requests for dispatch}

(One case is assumed if one case contains multiple patients.)

Figure 3

Percentage of duplicate requests for the Hokusoh DH (FY 2018). 


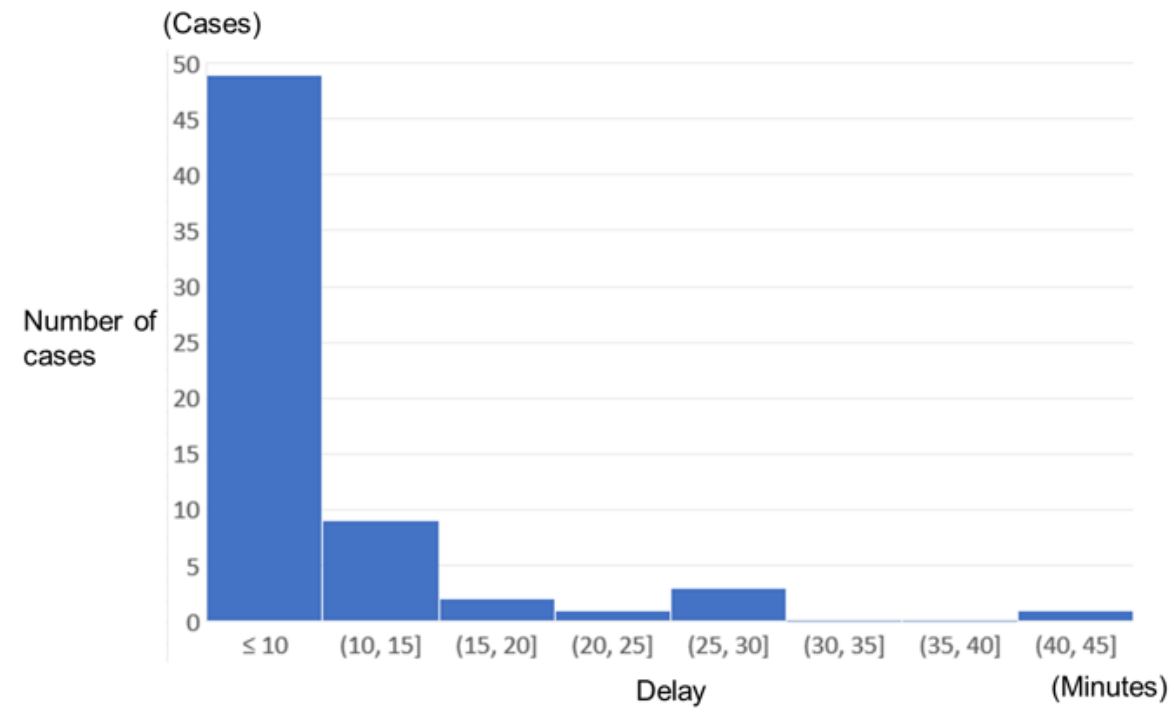

Figure 4

Delayed dispatch time for duplicate requests of the Hokusoh DH (FY 2018). 